\title{
Signal processing in optical coherence tomography for aerospace material characterization
}

\author{
Ping Liu \\ Roger M. Groves \\ Delft University of Technology \\ Optical Non-Destructive Testing Laboratory \\ Kluyverweg 1, 2600 GB, Delft, The Netherlands \\ E-mail: r.m.groves@tudelft.nl \\ Rinze Benedictus \\ Delft University of Technology \\ Structural Integrity \& Composites Group \\ Kluyverweg 1, 2600 GB, Delft, The Netherlands
}

\begin{abstract}
Based on a customized time-domain optical coherence tomography (OCT) system, a series of signal processing approaches have been designed and reviewed. To improve demodulation accuracy and image quality, demodulation approaches such as median filter, Hilbert transform, and envelope detector were investigated with simulated as well as experimental data. Without noise, the Hilbert transform has the best performance, but after considering the narrow-band noise in the modulated signal, the envelope detector was selected as the ideal demodulation technique. To reduce noise and enhance image contrast, digital signal processing techniques such as a bandpass filtering and two-dimensional median filtering were applied before and after the demodulation, respectively. Finally with integration of the customized OCT setup and designed signal processing algorithms, aerospace materials, such as polymer coatings and glass-fiber composites, were successfully characterized. The cross-sectional images obtained clearly show the microstructures of the materials. ( 2013 Society of Photo-Optical Instrumentation Engineers (SPIE) [DOI: 10 .1117/1.OE.52.3.033201]
\end{abstract}

Subject terms: optical coherence tomography; signal processing; demodulation; median filter; aerospace materials; microstructure.

Paper 121697P received Nov. 20, 2012; revised manuscript received Jan. 22, 2013; accepted for publication Feb. 8, 2013; published online Mar. 4, 2013.

\section{Introduction}

Optical coherence tomography (OCT) is a contactless and nondestructive image modality based on the principle of low coherence interferometry. It performs cross-sectional tomographic imaging of the microstructure within scattering media by measuring backscattered or backreflected light. Image resolutions of 1 to $20 \mu \mathrm{m}$ can be achieved, one to two orders of magnitude higher than with a conventional ultrasonic scan. ${ }^{1}$ Originally developed for the imaging of the human retina, ${ }^{2}$ these days OCT not only is widely used in the field of biomedical diagnostics, ${ }^{3-7}$ but it also shows a high potential in other applications, e.g., within the field of nondestructive testing (NDT). It has been shown that OCT is a promising NDT tool for the inspection of materials such as glass, paper, polymers, and ceramics. ${ }^{8-12}$ However, compared with biomedical counterparts, investigations so far are limited and the most recent advances in OCT imaging techniques, e.g., signal and image processing of OCT data, have not yet been extensively studied.

In a time-domain OCT (TD-OCT) system, the axial interferogram is modulated by the Doppler frequency shift induced by the movement of the reference arm. Typically, this demodulation is carried out in fixed electronics in an analog way. The general AM (amplitude modulated) demodulation method, mixing, is the technique used most often. ${ }^{13}$ It implements multiplication with a reference sinusoidal signal of the correct phase followed by low-pass filtering. However, sometimes this approach does not generate a perfect signal envelope owing to the variation of the carrier

0091-3286/2013/\$25.00 @ 2013 SPIE frequencies induced by nonlinear sweeping of the reference mirror. Digital signal processing techniques for OCT signal demodulation are rarely studied, and the only detailed publication that can be found is Ref. 14. Methods such as boxcar averaging, coherence, and Hilbert transform were investigated with simulated data in that paper. However, the authors did not consider the noise coupled in the signal, which can have a large influence on the accuracy of demodulation.

Therefore, this paper aims at developing a series of signal processing methods to improve demodulation accuracy and image quality. First, a customized OCT system for material characterization was built. Based on the OCT setup, a variety of demodulation approaches, e.g., median filter, Hilbert transform, and envelope detector, were investigated and evaluated. Denoising methods such as bandpass filter and two-dimensional (2-D) median filter were applied before and after the demodulation, respectively. Finally, with the integration of customized OCT setup and designed signal processing algorithms, specimens such as glass-fiber composites and polymer coatings were characterized. 2-D crosssectional images of these specimens are shown.

\section{Experimental Setup}

For our study, a compact and robust fiber-optic OCT system was built, as shown in Fig. 1. It uses a fiber-coupled lowcoherence superluminescent diode (SLD) (FESL-1550-20BTF, Frankfurt Laser, Germany). The laser source has a wavelength centered at $1550 \mathrm{~nm}$ with a full width at half maximum (FWHM) of $60 \mathrm{~nm}$, producing a theoretical axial resolution of $17.6 \mu \mathrm{m}$ in air. The light from the SLD, coupled with an optical circulator and fiber coupler 


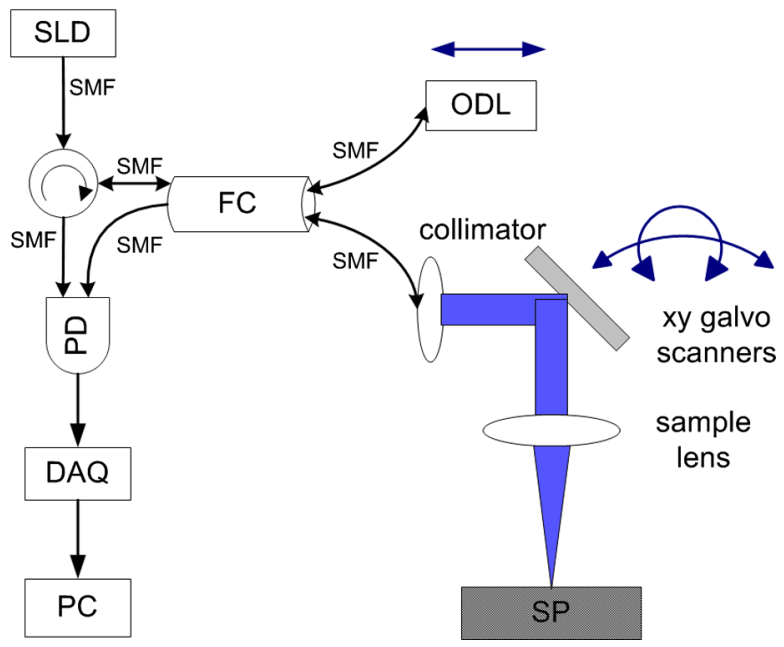

Fig. 1 Schematic setup of fiber-optic time-domain optical coherence tomography (TD-OCT). SLD, superluminescent diode; SMF, singlemode fiber; FC, fiber coupler; ODL, optical delay line; SP, sample; $\mathrm{PD}$, photo detector; $\mathrm{DAQ}$, data acquisition board.

(50:50), is divided into the sample and reference arms. Light backreflected from the sample and reference mirror is recombined and directed to the photo detector.

The axial scan (A-scan) in our OCT system is achieved by electric control of an optical delay line (ODL-650-MC, OZ Optics, Canada) in the reference arm. The servo motor in the ODL provides a linear resolution of $<1 \mu \mathrm{m}$ and can change the optical pathlength at a speed of $1.7 \mathrm{~mm} / \mathrm{s}$. In the sample arm, a $x y$-galvanometer scanner unit (GVS002, Thorlabs, Germany) is equipped to scan the sample transversely with a maximum scan frequency of $1 \mathrm{kHz}$. A balanced detector (PDB420C, Thorlabs) is also employed in the system to ensure a high signal-to-noise ratio (SNR). Finally the data is recorded by a PC with data acquisition board (NI 9225, National Instruments, US), and further processing is performed using the signal processing software described in the next section.

\section{Signal Processing}

In TD-OCT, a single axial profile of optical reflectivity within the sample is obtained by rapidly translating the pathlength of the reference arm and synchronously recording the magnitude of the resulting interference fringes. With a 50/50 beam splitter, the intensity collected by the photo detector $I_{d}$ can be expressed as: ${ }^{15}$

$I_{d}=0.5\left(I_{r}+I_{s}\right)+\operatorname{Re}\left[E_{r}^{*}(t+\tau) E_{s}(t)\right]$,

where $I_{r}$ and $I_{s}$ are the mean intensities returning from the reference and sample arms of the interferometer, which donate the DC components in the interference signal. The second term of the equation represents the amplitude of the interference fringes that carry information about sample structure. $E_{r}$ and $E_{s}$ correspond to optical fields from the reference and sample arms, respectively. $t$ is the time variation, and $\tau$ is the optical time delay set by the position of the reference mirror. Assuming that the sample and reference arms consist of uniform, linear, nondispersive material, the light source has a Gaussian power spectral density given by
$S\left(\omega-\omega_{0}\right)=\left(\frac{2 \pi}{\sigma_{\omega}^{2}}\right)^{1 / 2} \exp \left[-\frac{\left(\omega-\omega_{0}\right)^{2}}{2 \sigma_{\omega}^{2}}\right]$

where $\omega_{0}$ is defined as the center frequency and $2 \sigma_{\omega}$ is the standard deviation. Then the interference fringe, the second term in Eq. (1), can be described as

$I \propto \exp \left[-\frac{\sigma_{\omega}^{2} \Delta \tau_{g}^{2}}{2}\right] \cos \left(2 \pi \frac{2 v_{m}}{\lambda_{0}} t\right)$,

where $\Delta \tau_{g}$ is defined as the group delay, ${ }^{1}$ and $\lambda_{0}$ is the center wavelength of the light source. $v_{m}$ and $t$ are the velocity and duration of travel of the reference mirror, respectively. From Eq. (3) it can be seen that the amplitude of the interference fringe contains a Gaussian envelope and a cosine carrier with frequency

$f_{d}=\frac{2 v_{m}}{\lambda_{0}}$

which is given by the assumption of free space propagation. However, in an actual OCT system, there are two major obstacles for the acquisition of the Gaussian envelope that contains the structure information of the tested sample. First, the translation stages carrying the reference mirror are commonly available with several types of actuators such as DC motor, stepper motor, and server motor (used in our OCT system). All will have some degree of nonlinearity in their motion due to jitter and/or quantization of the scan motion (i.e., a stepper motor moves in discrete steps, giving approximately constant motion). Therefore, owing to the nonlinear sweeping of the reference mirror, the carrier frequency, also known as the Doppler frequency, is not perfectly constant but has small variations during the depth scan. Because the general AM demodulation requires a reference signal with same frequency and phase, it is not applicable for an OCT signal. The second obstacle is noise. Typically there are two kinds of noise in an OCT system. One is the random noise from the light source, the electronics, and the environment (e.g., mechanical vibration). The other is speckle noise, which originates from distorted wavefronts of light returning from sample that result in randomly appearing constructive and destructive features in the interferogram. ${ }^{16}$ These noise contributions are mixed and can acutely degrade the OCT image quality.

To extract the OCT envelope and improve the image quality, a succession of signal processing methods have been developed, as shown in Fig. 2. Once an axial signal was obtained, bandpass filtering was performed to reduce excess signal noise and remove the DC component. Then three different solutions were evaluated, and the optimum one for our OCT application was identified. After accumulating a series of processed axial signals, a cross-sectional image can be constructed. To reduce the speckle noise, 2-D image smoothing was applied before the final image was displayed.

\subsection{Bandpass Filter}

Although a balanced detector is used in the system to improve the SNR, residual noise that degrades the quality of OCT images still exists. Because the OCT signal is modulated by a carrier with the Doppler frequency, a bandpass 


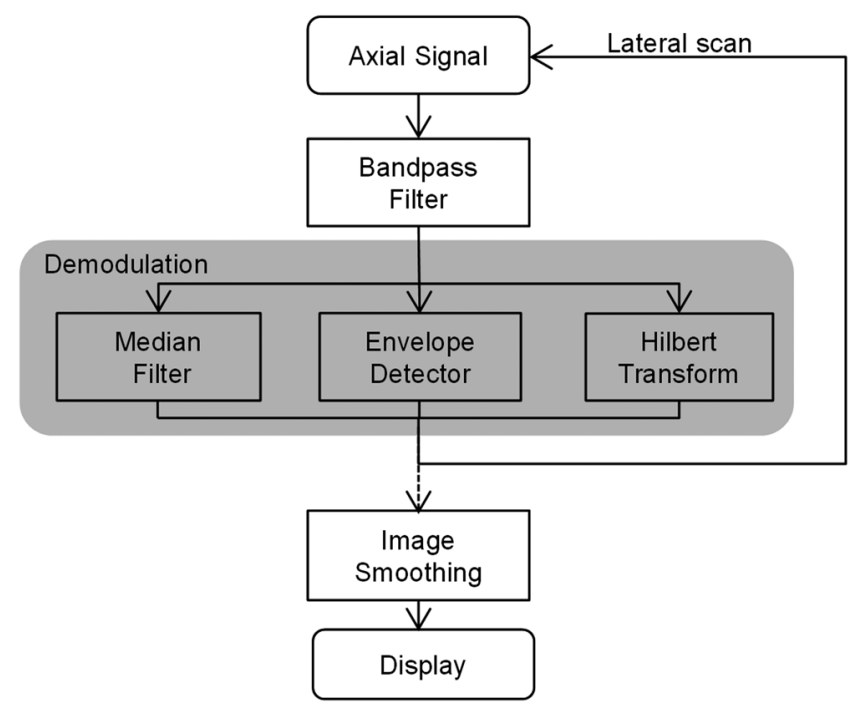

Fig. 2 Flowchart for signal/image processing of OCT data from the customized TD-OCT system.

filter can be used as the first step to remove both the noise and the DC component of the signal. For this application, a fourth-order Butterworth filter was designed. The center frequency of the bandpass filter is the Doppler frequency $f_{d}$. The FWHM of the filter is chosen initially to match the bandwidth of the light source, $\Delta \lambda$, using the following simple relationship:

$\mathrm{FWHM}=\frac{\Delta \lambda f_{d}}{\lambda}$

In practice, the filter bandwidth is chosen to be larger than the signal bandwidth. A filter bandwidth that is too narrow will widen the signal in time and arbitrarily limit the axial point spread function; ${ }^{17}$ however, a bandwidth that is too large will let in more noise and reduce the minimum detectable reflectivity or dynamic range of the system. Here, twice the signal bandwidth was selected, and the magnitude response of this filter is shown in Fig. 3.

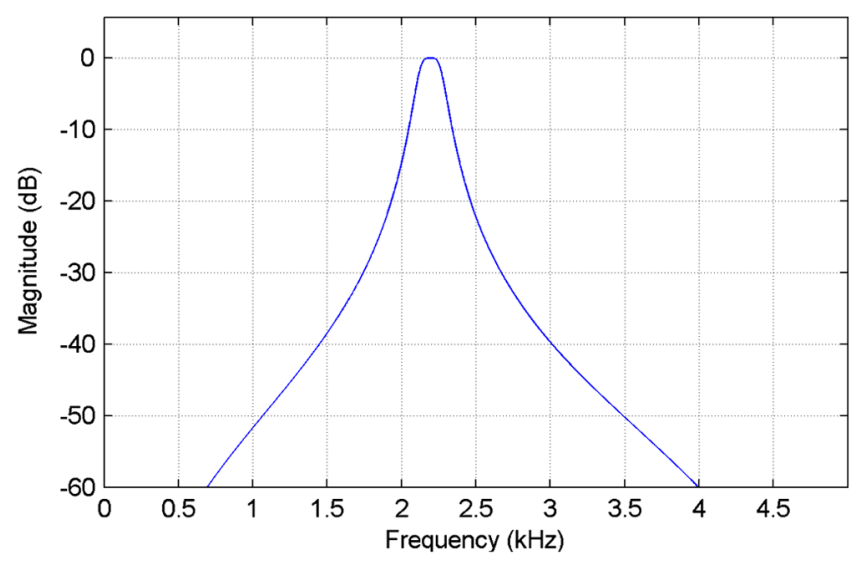

Fig. 3 Magnitude response function for a Butterworth bandpass filter. The center frequency and the bandwidth are 2193 and $170 \mathrm{~Hz}$, respectively.

\subsection{Demodulation}

After bandpass filtering, the axial OCT signal can be simply expressed by

$x[n]=m[n] \cos (\omega[n] n+\phi[n])$,

where $m[n]$ is the "message signal" of interest and $\cos (\omega[n] n+\phi[n])$ is the carrier. As discussed above, owing to the nonlinear sweeping of the reference mirror, the frequency $\omega[n]$ of the carrier has small variations, which makes the general AM demodulation unsuitable. Therefore three demodulation methods that are largely insensitive to variations in the carrier frequency have been investigated. Both simulated and experimental data were used to evaluate the accuracy of the three methods.

The first technique, a median filter, is not a true demodulation method but is extremely rapid due to its low computational complexity. In this method, the envelope can be extracted by collecting the median of the absolute value of the modulated signal in the sliding window. However, the accuracy is quite sensitive to the choice of window length, which is the main drawback of this method.

The second technique, Hilbert demodulation, is based on the decomposition of the modulated signal into two components. After a Hilbert transform, a purely real signal $x[n]$ can be expressed as the sum of two complex signals:

$x[n]=x_{a}[n]-j x_{h}[n]$,

where $x_{a}[n]$ and $x_{h}[n]$ represent the separated analytic and Hilbert signals, respectively. Because $x[n]$ can be expressed in complex notation as Eq. (6), the analytic and Hilbert signals can be calculated as

$x_{a}[n]=\frac{1}{2} m[n] e^{j(\omega[n]+\phi[n])}$

and

$x_{h}[n]=-\frac{1}{2} m[n] e^{-j(\omega[n]+\phi[n])}$.

Therefore message $m[n]$ can be recovered by taking twice the absolute value of either the analytic signal or the Hilbert signal.

The third technique, an envelope detector, typically consists of a rectifier and a lowpass filter in analog circuits. In software, it is easy to realize by lowpass filtering the autocorrelation of the modulated signal. Here the autocorrelation function can be expressed as

$x[n] * x[n]=\frac{1}{2} m^{2}[n]+\frac{1}{2} m^{2}[n] \cos (2 \omega[n] n+2 \phi[n])$.

After autocorrelation, it can be seen that half the energy of the modulated signal is pushed up to higher frequencies and half is shifted down toward DC. Message $m[n]$ can be recovered by a lowpass filter and the square root function.

To evaluate the accuracy of the three demodulation techniques, a simulated OCT signal with a narrowband noise was tested. According to Eq. (6), the message signal $m[n]$ was specified as a Gaussian pulse with a peak located in the middle of the signal. Three separate carrier frequencies 


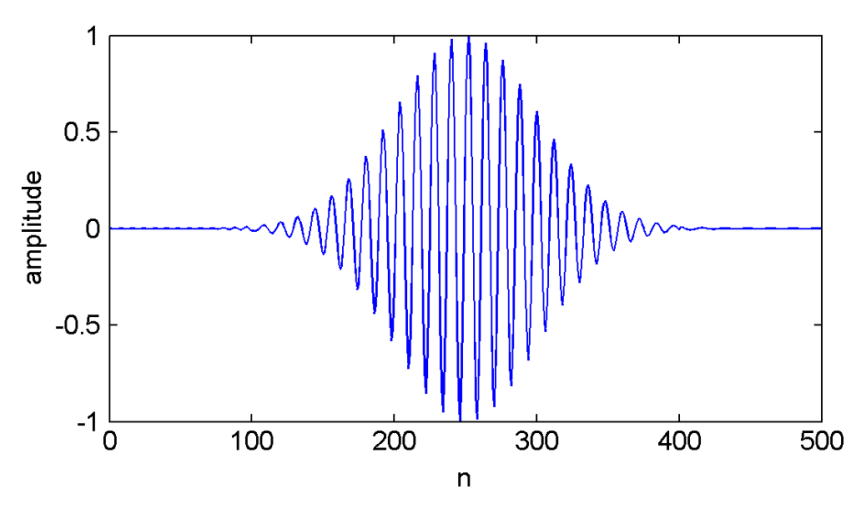

Fig. 4 Simulated OCT signal without noise.

$(\omega[n]=\pi / 8, \pi / 6$, and $\pi / 4)$ were present consequently during the whole period. The simulated signal is shown in Fig. 4. To be closer to an actual OCT signal, $\xi[n]$, a narrowband noise with a power specified in the range $0 \%$ to $25 \%$ of the modulated signal was added. It was acquired by bandpass filtering of the white noise, where the design of the filter follows the principles mentioned in Sec. 3.1 (a fourth-order Butterworth filter with a bandwidth from $\pi / 16$ to $5 \pi / 16$ ). To quantitatively compare the three methods, the mean square error (MSE) between $m[n]$ and the demodulated message $\hat{m}[n]$ was calculated as

$\mathrm{MSE}=\frac{1}{N} \sum_{n=0}^{N-1}(m[n]-\hat{m}[n])^{2}$.

Considering the time delay after lowpass filtering within the envelope detector, a fourth-order finite impulse response (FIR) filter with Hanning window was selected, providing a constant group delay with a value of 2 . For calculating the MSE, the data after the envelope detector was shifted by 2 and the end of the simulated signal (which was longer than used in MSE calculation) was cut.

Figure 5 shows how the MSE of each algorithm varies as a function of the noise percentage added in the simulated data. It can be seen that although the optimized window length ( 25 points each) is chosen, the method with a median filter gives the worst accuracy except in the high noise range, $>20 \%$. The results from the two other methods are quite

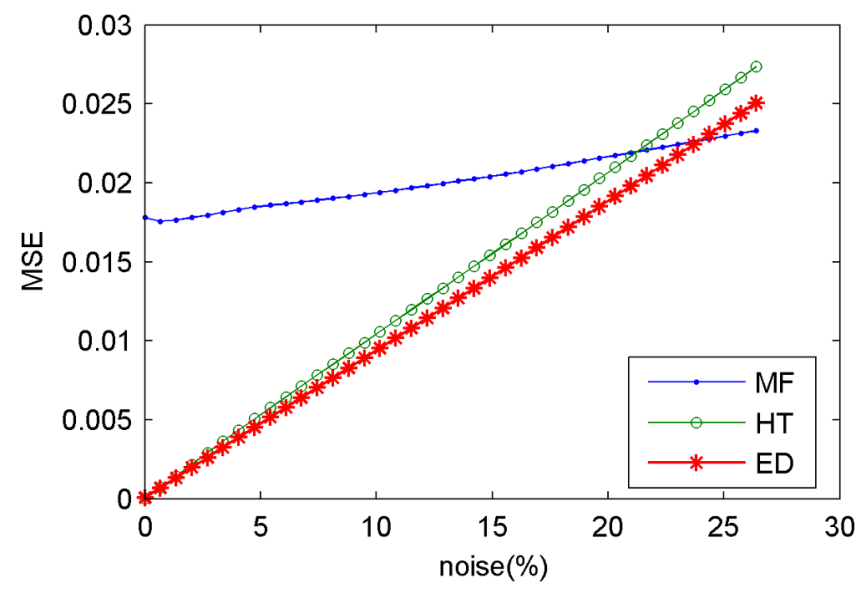

Fig. 5 Mean square error (MSE) versus noise percentage for various demodulation algorithms applied to simulated OCT data. MF, median filter; HT, Hilbert transform; ED, envelope detector.
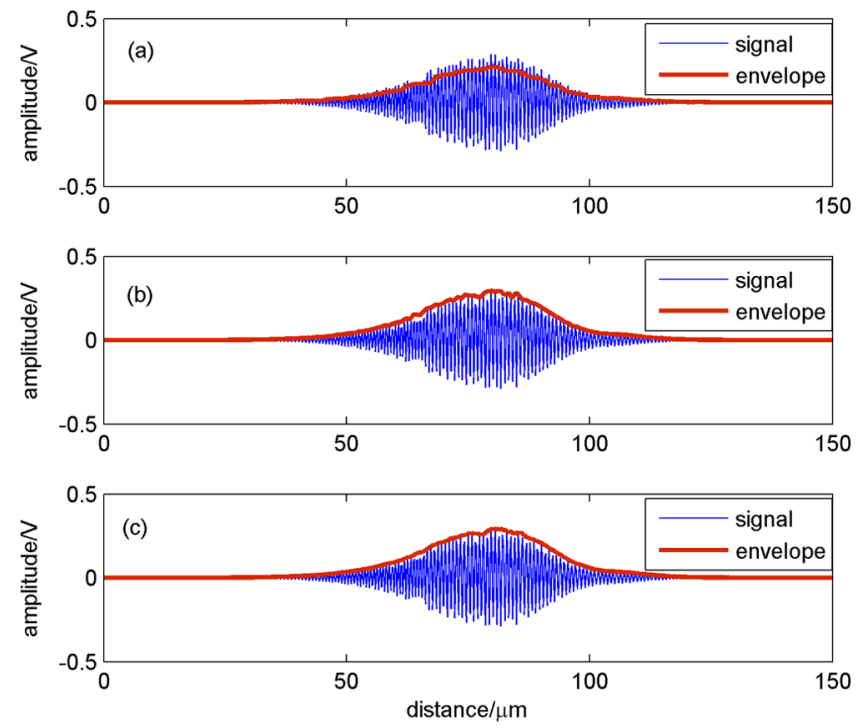

Fig. 6 Demodulation of experimental signal by median filter (a), Hilbert transform (b), and envelope detector (c). The window size for median filter is optimized to 25 . The filter in the envelope detector is a fourth-order Hanning window finite impulse response (FIR) filter.

similar at the very first phase when the noise is $<2 \%$. However, with an increase of the noise, the method with envelope detector gives better performance than that with Hilbert transform owing to the benefit from the lowpass filter, which removes the high-frequency noise from the envelope.

Experimental data was also used to evaluate the three methods by using a reflecting mirror as the sample. After bandpass filtering, the signal is shown in Fig. 6. The red lines in each image of the figure are the envelopes extracted by the three algorithms. Similar to the results from the simulated data, the performance of envelope detector and Hilbert transform is better than that of the median filter. The envelope extracted by envelope detector is smoother than that by Hilbert transform. Therefore the envelope detector was selected as the demodulation method to match with our OCT setup.

\subsection{2-D Smoothing}

Like other coherent image techniques, OCT suffers from speckle noise that degrades the image quality. Therefore, speckle noise removal is a critical preprocessing step for image enhancement. In this paper, a 2-D median filter was used for smoothing the cross-sectional image. The value of each pixel was replaced by a median value in a local neighborhood (a $N \times N$ window where $N=3,5,7$, etc.). The experimental results are shown in the next section.

\section{Results and Application}

In this paper, two aerospace materials, an epoxy coating and a glass-fiber reinforced polymer (GFRP) composite, were tested. Epoxy coatings are widely employed for corrosion protection of steel pipes and metal containers for gas, oil, and water, whereas GFRP composites are extensively applied for the production of aerospace parts such as helicopter rotor blades. Because of their semitransparent property, OCT could be an ideal tool to inspect the internal structures of these materials. 


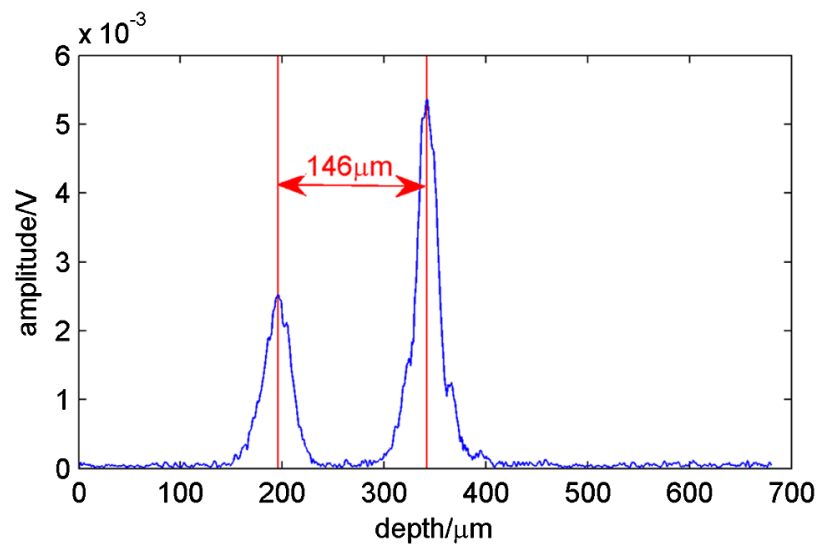

Fig. 7 Envelope of a single depth scan signal from a good-quality epoxy coating.

\subsection{Epoxy Coatings}

In our experiment, the epoxy coating used is a transparent Resolution Epikote 1001 coating with a thickness of $95 \pm 5 \mu \mathrm{m}$. The substrate is a $99.5 \%$ pure AA1050 aluminum alloy with surface treatment. For a comparative analysis, two samples with different quality were selected: a sample with a healthy coating and one with substrate defects.

Figure 7 demonstrates the envelope of one depth scan signal from an epoxy sample with good quality. The two peaks in the figure show the strong reflections from the front and bottom surfaces. The corresponding optical scan distance is $146 \mu \mathrm{m}$. Taking 1.55 as the refractive index of the epoxy layer, ${ }^{18}$ the calculated physical thickness is $94.2 \mu \mathrm{m}$. Compared with $97.5 \mu \mathrm{m}$ measured by a thickness gauge (PosiTector 6000, DeFelsko, US), the result is acceptable considering the $17.6-\mu \mathrm{m}$ depth resolution provided by the SLD in our setup.

Figure 8 shows the cross-sectional images from the sample with substrate defects. Similar to the results shown in Fig. 7, the first two dark lines in Fig. 8 indicate the positions of front and back surfaces of the epoxy coating. However, the difference is that an additional interference (a third dark line in Fig. 8) emerges underneath the coating layer with a depth of around $250 \mu \mathrm{m}$, identifying a prefabricated hole introduced to the substrate surface.

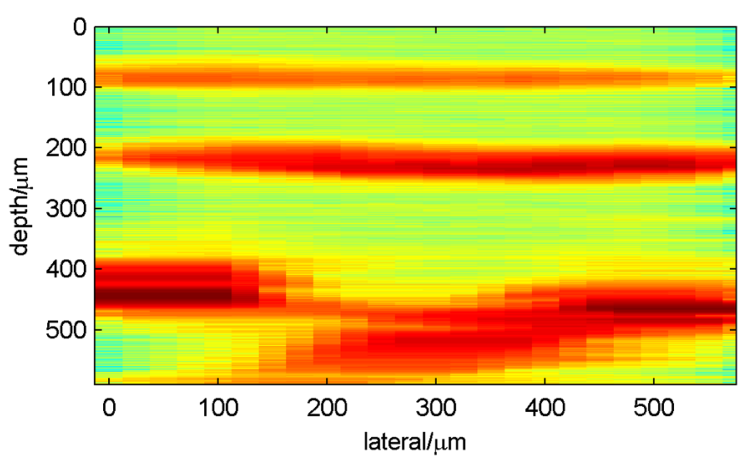

Fig. 8 Cross-sectional image from the coating sample with substrate defects.

\subsection{Glass-Fiber Composites}

Another specimen investigated is a GFRP (PP-GF70, Ticona, US), a $70 \%$ by weight endless fiber-reinforced unidirectional polypropylene tape. Figure 9(a) shows the original cross-sectional image of the glass-fiber composite. The fiber layers are observable as dark lines in between the matrix. However, the speckle degrades the quality of the image. Figure 9(b)-9(d) is the enhanced images by 2-D median filter with different window size. It can be seen that the image contrast is substantially improved and the structure of the sample is much more obvious. However, it is also worth noting that as the window size increases, the image resolution is decreased and some detail of structural information is lost. Because it is preferable to reduce the speckle while at the same time preserving the structural information, an intermediate window size of $7 \times 7$ pixels has been selected for this application.

\section{Discussion}

The extraction of each axial envelope signal is the first important step that decides the quality of the OCT image. In this paper, three demodulation techniques, median filter, Hilbert transform, and envelope detector, were investigated. A median filter has minimum computational complexity but the least accuracy when the modulated signal contains low noise ( $20 \%$ by power compared with the signal from the simulation). Hilbert transform can give better performance but is quite sensitive to noise. The MSE value increases dramatically with the noise growth. The ideal one is envelope detector, which is realized by lowpass filtering the autocorrelation of the modulated signal. At the very beginning ( $<2 \%$ noise), the accuracy of envelope detector is even a little bit lower than that of Hilbert transform due to the influence of the filter (e.g., passband ripple and stopband attenuation). However, it shows an advantage as the noise level increases because the lowpass filter in the envelope detector can remove the highratio noise and guarantee the demodulation accuracy.

Of equivalent importance in signal processing is noise reduction. Image averaging is a widely used technique to suppress random additive noise. However, it is not suitable for TD-OCT image processing because of its low efficiency caused by repeated scanning. In our design, a bandpass filter and 2-D median filter were used before and after the demodulation, respectively. The bandpass filter acted as signal preprocessing to remove the noise and DC component that can largely affect the result of demodulation. The 2-D median filter was applied as the final step for further image enhancement.

With the designed signal processing methods, two materials, epoxy coating and GFRP composite, were investigated as shown in Figs. 7-9. Compared with our previous studies on the same materials but using Hilbert transform solely without further enhancement, ${ }^{19}$ the quality of the images presented here are greatly improved and the microstructure of the materials is more clearly shown. In addition, Fig. 9 shows the tradeoff between the resolution and speckle reduction using 2-D median filter with increased window. To date, only limited research has been done in the area of image enhancement and speckle reduction in OCT imaging. ${ }^{20-23}$ Most techniques that have been developed trade resolution for reduction of speckle contrast. Further work in this area is needed. 
(a)

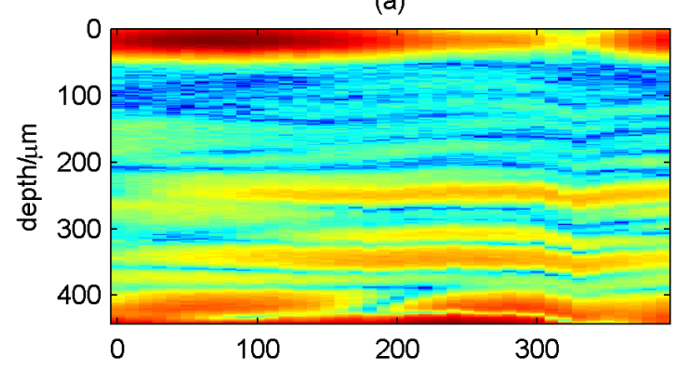

(c)

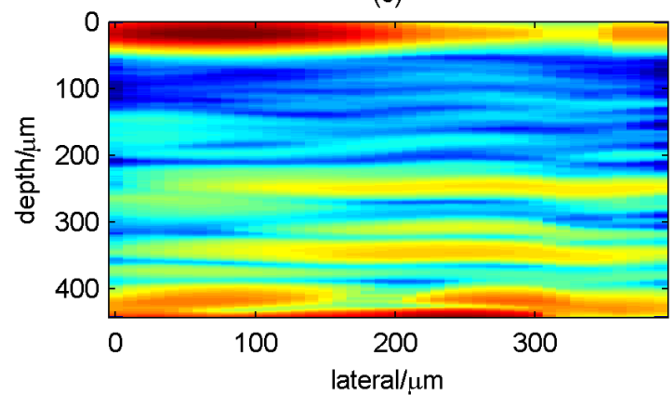

(b)

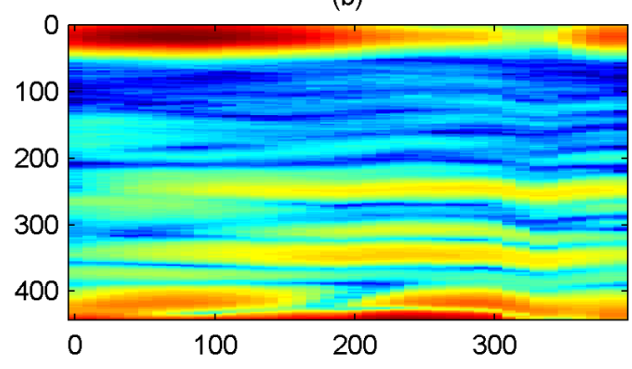

(d)

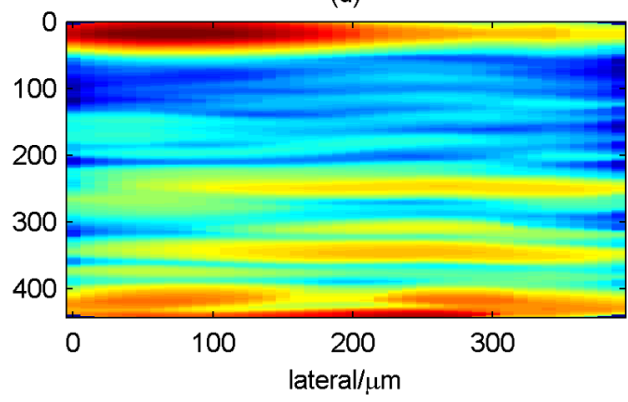

Fig. 9 Cross-sectional images taken from a unidirectional tape made of GFRP. (a), Original OCT image. Processed by 2-D median filter with window size $3 \times 3(b), 7 \times 7$ (c), and $11 \times 11($ d).

\section{Conclusions}

This study explored simple but highly effective methods for envelope detection and image enhancement in a TD-OCT system. After comparison, the envelope detector was selected as the ideal method for the demodulation of each axial signal. A bandpass filter and 2-D median filter were applied before and after the demodulation, respectively, to reduce random noise and speckle noise. Two samples of aerospace materials, epoxy coating and glass-fiber composite, were tested. The cross-sectional images clearly show the microstructure of the materials. The quality of the sample, e.g., thickness, homogeneity, and defects, can be easily evaluated. These initial results are encouraging and suggest that a combination of bandpass filter, envelope detector, and median filter could play an important role in the signal postprocessing for the reconstruction of the microstructure within materials.

\section{References}

1. B. E. Bouma and G. J. Tearney, Handbook of Optical Coherence Tomography, Marcel Dekker, New York (2002).

2. D. Huang et al., "Optical coherence tomography," Science 254(5035), 1178-1181 (1991).

3. J. A. Izatt et al., "Optical coherence tomography and microscopy in gastrointestinal tissues," IEEE J. Sel. Top. Quant. Electron. 2(4), 1017-1028 (1996).

4. A. Baumgartner et al., "Polarization-sensitive optical coherence tomography of dental structures," Caries Res. 34(1), 59-69 (2000).

5. J. G. Fujimoto, "Optical coherence tomography for ultrahigh resolution in vivo imaging," Nat. Biotechnon. 21(11), 1361-1367 (2003).

6. D. C. Adler et al., "Three-dimensional endomicroscopy using optical coherence tomography," Nat. Photon. 1(12), 709-716 (2007).

7. W. Drexler and J. G. Fujimoto, "State-of-the-art retinal optical coherence tomography," Prog. Retin. Eye Res. 27(1), 45-88 (2008).

8. M. Bashkansky et al., "Subsurface defect detection in ceramics by highspeed high-resolution optical coherent tomography," Opt. Lett. 22(1), 61-63 (1997).

9. J. Jasapara, "Non-invasive characterization of microstructured optical fibers using Fourier domain optical coherence tomography," Opt. Express 13(4), 1228-1233 (2005).
10. E. Alarousu et al., "Study on the use of optical coherence tomography in measurements of paper properties," Meas. Sci. Tech. 16(5), 1131 (2005).

11. K. Wiesauer et al., "En-face scanning optical coherence tomography with ultra-high resolution for material investigation," Opt. Express 13(3), 1015-1024 (2005).

12. D. Stifter, "Beyond biomedicine: a review of alternative applications and developments for optical coherence tomography," Appl. Phys. B 88(3), 337-357 (2007).

13. M. E. Brezinski, Optical Coherence Tomography: Principles and Applications, Academic Press, New York (2006).

14. D. C. Adler, Digital Signal Processing Techniques for Optical Coherence Tomography: OCT and OCT Image Enhancement, Massachusetts Institute of Technology, Cambridge, Massachusetts (2004).

15. J. M. Schmitt, "Optical coherence tomography (OCT): a review," IEEE J. Sel. Top. Quant. Electron. 5(4), 1205-1215 (1999).

16. J. M. Schmitt, S. Xiang, and K. M. Yung, "Speckle in optical coherence tomography," J. Biomed. Opt. 4(1), 95-105 (1999).

17. M. R. Hee, Optical Coherence Tomography of the Eye, Massachusetts Institute of Technology, Cambridge, Massachusetts (1997).

18. J. E. Mark, Physical Properties of Polymers Handbook, Springer, New York (2006).

19. P. Liu, R. M. Groves, and R. Benedictus, "Quality assessment of aerospace materials with optical coherence tomography," Proc. SPIE 8430 , 843001 (2012).

20. M. Bashkansky and J. Reintjes, "Statistics and reduction of speckle in optical coherence tomography," Opt. Lett. 25(8), 545-547 (2000).

21. J. Rogowska and M. E. Brezinski, "Image processing techniques for noise removal, enhancement and segmentation of cartilage OCT images," Phys. Med. Biol. 47(4), 641 (2002).

22. M. Pircher et al., "Speckle reduction in optical coherence tomography by frequency compounding," J. Biomed. Opt. 8(3), 565-569 (2003).

23. D. C. Adler, T. H. Ko, and J. G. Fujimoto, "Speckle reduction in optical coherence tomography images by use of a spatially adaptive wavelet filter," Opt. Lett. 29(24), 2878-2880 (2004).

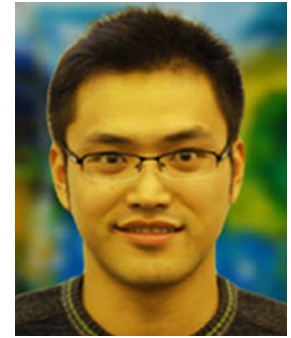

Ping Liu is a PhD candidate in optical non-destructive testing from Delft University of Technology. He received BS and MS degrees in mechanical and electrical engineering from Nanjing University of Aeronautics and Astronautics, China, in 2008 and 2010 , respectively. His research is focused on optical coherence tomography for aerospace material characterization. 


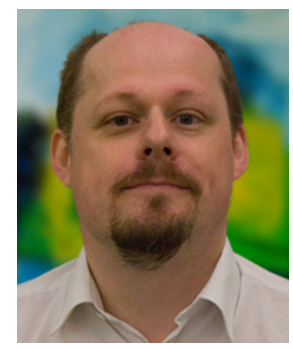

Roger M. Groves is assistant professor and head of the Optical Non-Destructive Testing Laboratory in the Faculty of Aerospace Engineering at Delft University of Technology. He has a PhD in optical sensors from the School of Engineering, Cranfield University, United Kingdom, an MSc degree in applied physics from the University of Hertfordshire, United Kingdom, and a BSc degree in chemistry from the University of Wales, Aberystwyth. His research interests are in optical instrumentation for non-destructive testing and strain measurement, applied to engineering and cultural heritage applications. He has developed novel instrumentation based on shearography, digital holography, optical fiber Bragg gratings, Moiré, and digital image correlation and this is supported by more than 50 publications in this field.

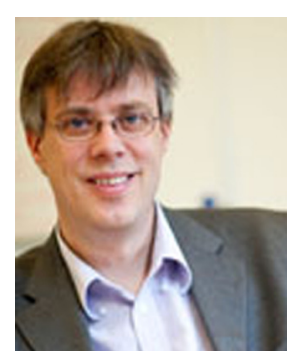

Rinze Benedictus is a professor and head of the Structural Integrity \& Composites Group head of the Department of Aerospace Structures and Materials, and assistant dean of the Faculty of Aerospace Engineering, Delft University of Technology. He has an MSc degree in applied physics and a $\mathrm{PhD}$ in materials science. He has more than 30 patents assigned to his name and since 2005 has published more than 40 publications in the field of aerospace materials and structures, with an emphasis on damage and structural integrity. 